\title{
MODELLING OF TEXTURE DEVELOPMENT DURING NUCLEATION AND GRAIN GROWTH
}

\author{
Ph. Tavernier and J.A. Sypunar \\ Department of Mining and Metallurgical Engineering \\ MoGill University, Montreal \\ H3A 2 A7 Canada
}

\begin{abstract}
A model of development of recrystallization texture has been proposed based on physical processes observed during nucleation and growth of grains. In this model the nucleation is driven by energies stored in various texture components. The assumption is made that there exists a specific relationship between the place the nucleation starts and the orientation of the nuclei. The grain growth modelling is determined by the grain boundary energy and is related to assumptions that certain orientations of grain are favoured during the grain growth.

The model is used to simulate the texture development in low-carbon steel and offers in addition to textural information the prediction of the kinetics of nucleation and grain growth illustrating the fundamental aspects of grain topology in the deformed and annealed metals which at the same time are general enough to allow to introduce in the future more realistic assumptions for nucleation and grain growth processes.
\end{abstract}

\section{MONTE-CARLO SIMULATION}

This texture model is inspired by the Monte-Carlo simulation of the grain growth proposed and used by Anderson and others [1-3] and is based on a similar two-dimensional matrix description of the microstructure. Accordingly, the microstructure is described by a matrix; each element of this matrix is called a site and represents a small area of the microstructure. Orientation of the site is given by a spin, which takes integer values corresponding to various texture components. Neighbouring sites with the same spin belong to the same grain. Each grain is therefore characterized by its size, obtained by counting the number of sites within it, and its orientation.

To simulate texture development several steps are taken; first of which is the generation of the deformed microstructure. The next step is the determination of the distribution of energy stored in this microstructure. The knowledge of this distribution allows for the determination of the nucleation sites. The nuclei grow until they reach a critical size, at which time different rates goveming the growth stage are introduced.

We will now illustrate various stages of texture development using the example of low- carbon steel.

\section{TEXTURE OF THE DEFORMED MITAL}

Having introduced a general principle used by us to define the microstructure, we need to describe how the structure and texture of deformed metal is introduced into the model. The microstructure should contain the information about the main features of deformed metals; among them are the degree of cold rolling, the grain size, the grain shape and the texture.

The rolling type of microstructure is obtained by deforming the isotropic grain shape, so that the grain shape changes follow the macroscopic change in the shape of the specimen. It is also assumed that the misorientation between subgrains belonging to the same grain is related to the degree of deformation. Such misorientation is obtained from known experimental data. Texture is introduced in a form of a finite set of orientations, such that for instance, orientation 1 represents one ideal texture, orientation 2 represents another texture and so forth. This representation is illustrated in Table 1 for low-carbon steel. Since normally the texture is represented by a continuous function (ODF), the discrete representation implies a loss of information and was adopted to simplify the computer calculation. Each ideal orientation is split into several orientations to simulate the observed spread of grain orientation around ideal texture. 


\section{TEXTURE DEVELOPMENT DURING NUCLEATION STAGE}

Stored energy of deformation is related to grain orientation and it is generally agreed that the nucleation process takes place in that area of the deformed metal where the highest energy is stored. This factor is considered a most important one affecting the competition between nuclei generated in grains of different orientation.

Stored energy of deformation in low-carbon steel is responsible for a sequence of nucleation events which according to Dillamore [4] and Hutchinson [5] correspond to the following relationship between the stored energy of deformation of different texture components.

\section{$\mathrm{U}(110)<110>\mathrm{U}(111)<\mathrm{UVW}>\mathrm{U}(211)<011>\quad \mathrm{U}(100)<011>$}

Dillamore [4] has pointed out that the variation of stored energy is related to a variation of the Taylor factor, therefore, this factor was used by us to calculate the energy stored in various texture components.

An exemplary topographic representation of the stored energy in $70 \%$ cold rolled low-carbon steel is represented in Fig.1 according to the texture data given in Table 1.

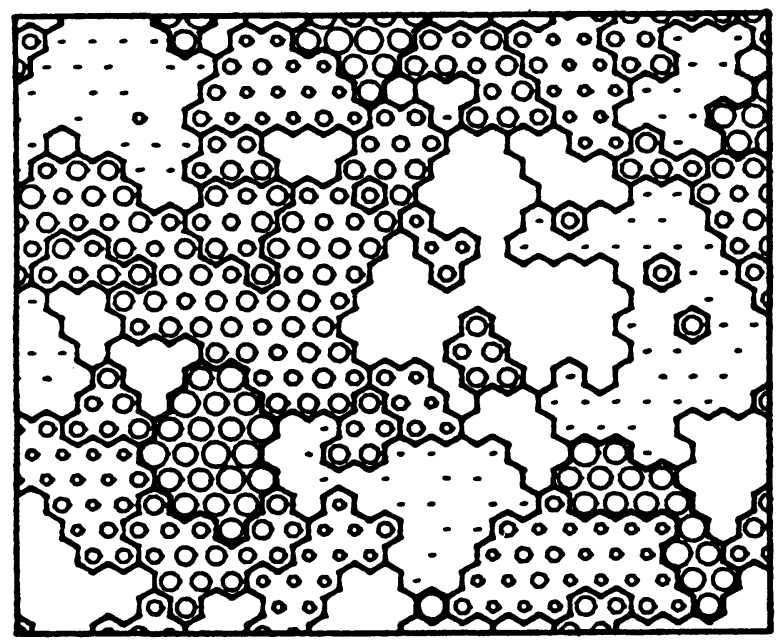

\section{legend}

$u<2.2$
$-2.2<U<2.8$
- $2.8<U<3.0$
- $3.0<U<3.4$
- $3.4<U<3.8$
$03.8<U<4.2$
$04.2<U<4.6$
$04.6<<10$.

Figure 1 An example of an energy map showing how texture and the misorientation inside of the grain affects the energy distribution.

Such an energy map is searched out using Monte-Carlo technique to identify the area of the deformed metal where the highest energy of deformation is stored and where high local misorientation between subgrains exists; these places are then considered to be potential nucleation sites.

There are also other factors introduced to simulate the nucleation behaviour. The first one is the level of analysis (LA) which is to account for the nucleation incubation period. The level decreases with time, and new texture starts to nucleate only when the local stored energy is lower than the level of analysis. If LA is zero, all texture components start to nucleate right at the beginning with a steady nucleation rate. If LA is not equal to zero the textures nucleate one after another according to the energy stored in each grain.

Another parameter, the transition band level, is introduced to simulate the nucleation at the transition bands.

If the flip occurs, the stored energy of the site is set to zero and the question arises about what will be the new orientation of the site. The answer to this question will determine the texture developed in the nucleation stage. For low-carbon steel, it has been assumed that the grain boundary sites have the same orientation as the deformed matrix while for the intemal sites there is a random selection of the new orientation among the orientations which are introduced in the simulation. This assumption agrees with the experiments [7] which demonstrated that the nuclei at transition bands often have random orientation. 
Table 1 Abundance of various texture components in low-carbon steel rolled $70 \%$.

$\begin{array}{cccc}\text { Texture } & \text { Abundance } & \text { Associated orientations } \\ (100)\langle 011\rangle & 15 \times & 1 \text { to } 15 \text { in } 5 \text { eroups } \\ (112)\langle 011\rangle & 30 \times & 16 \text { to } 15 & n \\ (111)\langle 011\rangle & 20 \times & 46 \text { to } 65 & n \\ (111)\langle 211\rangle & 15 \times & 66 \text { to } 80 & n \\ (011)\langle 011\rangle & 5 \times & 81 \text { to } 85 & n \\ \text { Random } & 5 \times & 86 \text { to } 90 & n \\ \text { Randon } & 5 \times & 91 \text { to } 95 & n \\ \text { Random } & 5 \times & 86 \text { to } 100 & n\end{array}$

Following these principles we simulate the kinetics of nucleation, topological evolution of the microstructure and texture evolution.

There are several parameters which effect the simulation; for instance, the transition band level, the grain size and the previous deformation. These parameters affect the distribution of nucleation sites and therefore influence the simulation by changing a probability of nucleation within the grain and also at the grain boundary.

Various simulations were performed to determine the effect of model parameters on the population of nuclei in various classes. Fig.2 summarizes the topological results of simulation and allows us to make several observations about the importance of various parameters on distribution of nucleation sites.

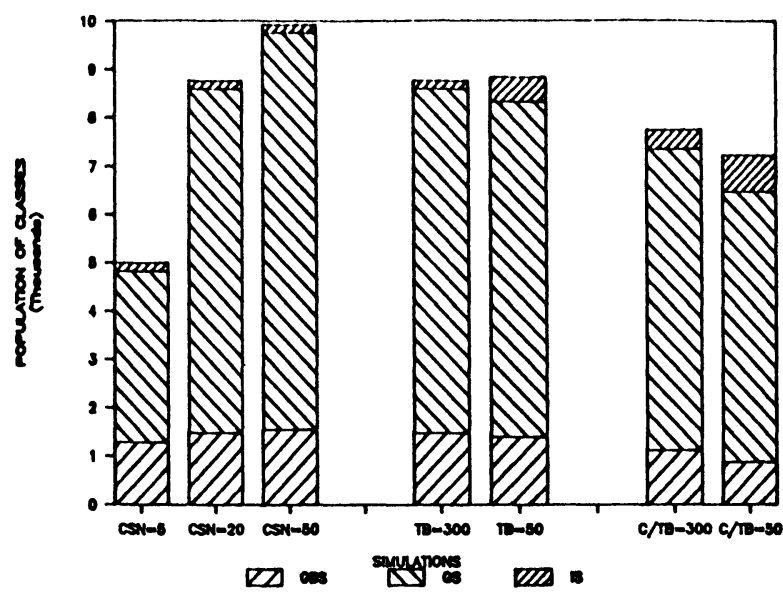

Figure 2 Analysis of nucleation sites. The population of the three classes are presented for different critical size of nuclei (5,20 and 50) and two microstructures, one with 18 sites per grain and another with 45 sites per grain. 
Typical microstructure illustrating the distribution of nucleating sites in the deformed matrix is presented in Fig.3.

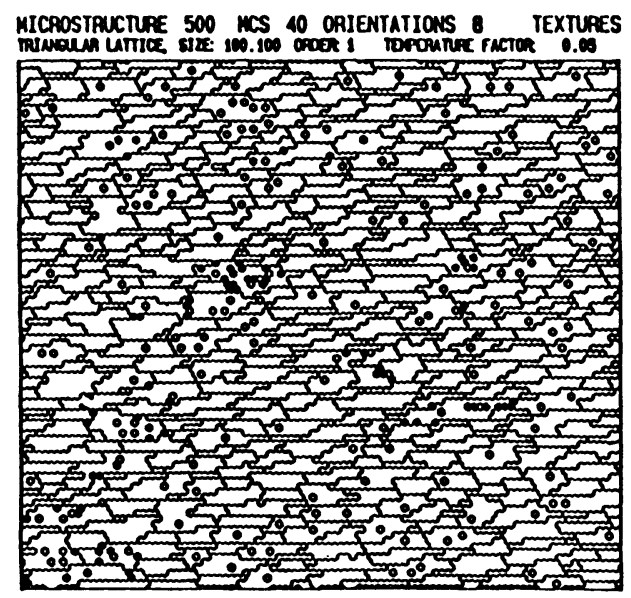

Figure 3 Example of microstructure obtained after 5 Monte-Carlo steps of simulation, showing various nucleation sites.

An exemplary texture developed during nucleation stage in cold rolled $70 \%$ steel is given in Fig.4.

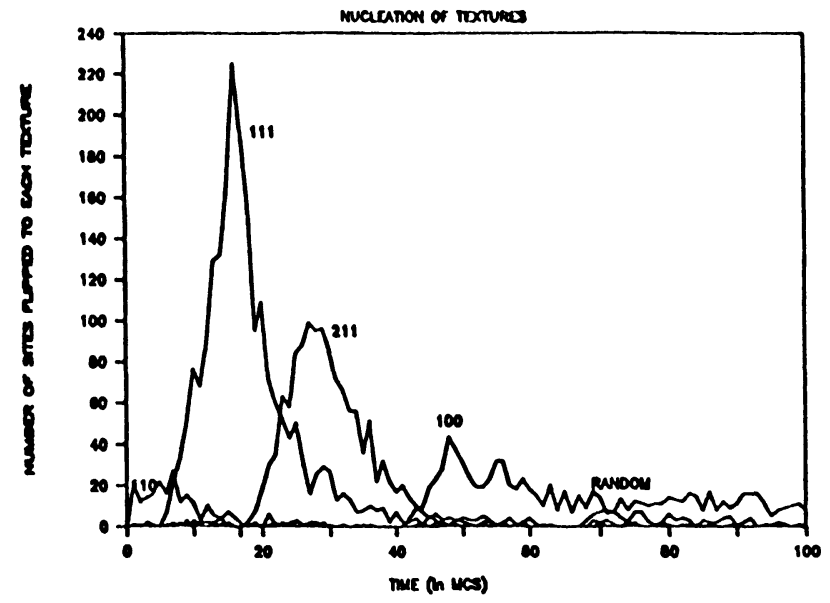

Figure 4 Result of simulation of nucleation of various texture components in low-carbon steel.

The high energy components of texture form the first nuclei in the deformed microstructure. Such nuclei will start growing at the expense of other grains and this will affect the development of texture. The domination of the nucleation sites at the grain boundary leads to a retention of texture represented in the deformed matrix. Both the increase in number of intemal sites at the transition band and the increase of the initial grain size favour the textures which are only weakly represented in the deformed matrix. The nucleation at the transition bands favour the development of textures only weakly represented in the deformed steel, and is for instance responsible for an increase in (110) texture components.

The texture development illustrated by the results obtained for low-carbon steel gives results which are very similar to ones reported by Hutchinson. This type of Monte-Carlo simulation of nucleation of texture will be 
now complemented by the simulation of the grain growth.

\section{TEXTURE DEVELOPMENT DURING GRAIN GROWTH}

A Monte-Carlo simulation technique has been extended to model the grain growth. The growth consists of a sequence of attempts to reorient sites, the success of which is determined by whether the conditions imposed by the energy of the grain boundary are satisfied.

The model relies on the definition of the grain boundary energy table. These data are not always known and were obtained using a limited experimental knowledge of the subject.

In order to determine the grain boundary energy, we analyze the neighbourhood of the chosen site up to the $n^{\text {中 }}$ order and add the contributions from each elementary boundary between the analyzed site and its neighbours, VPOT (aiaj). The grain boundary energy, E, can be expressed by the formula:

$$
\mathrm{E}_{i}=\sum_{j}^{\text {an }} \text { VPOT }\left(a_{i} a_{j}\right)\left(\delta\left(a_{i} a_{j}\right)-1\right)
$$

where $a_{i}$ - is a spin of the analyzed site $i$, and $a_{j}$ is the spin of the neighbour site. This description of the energy implies that if VPOT $(a, a)=0$, both grains have the same orientation. If for instance the growth of orientation " $a$ " versus orientation " $b$ " is favoured, then VPOT $(a, b)$ must be less than VPOT $(b, a)$.

The simulation of recrystallization texture requires the use of two different procedures, one for the nucleation and one for the grain growth. The grain boundary concept which originally applies to recrystallized grains only has been extended to treat all sites.

Following that, for the grain boundary between one deformed state and another deformed state, no motion is expected. Similarly, the deformed state cannot grow at the expense of the nuclei and, the deformed matrix cannot be consumed by the recrystallized grains.

Such a link between the nucleation and grain growth allows us to follow both processes simultaneously. The growth rate depends on several factors and can be changed using growth parameter which is a ratio between the nucleation rate and the growth rate. This parameter allows us to assign various levels of importance to different processes of texture development during recrystallization.

The final result of the texture simulation is illustrated in Fig.5 where evolution of texture in low-carbon steel annealing is shown. A development of a strong (111) component is observed while the $\{112\}$ and $\{100\}$ texture components disappear.

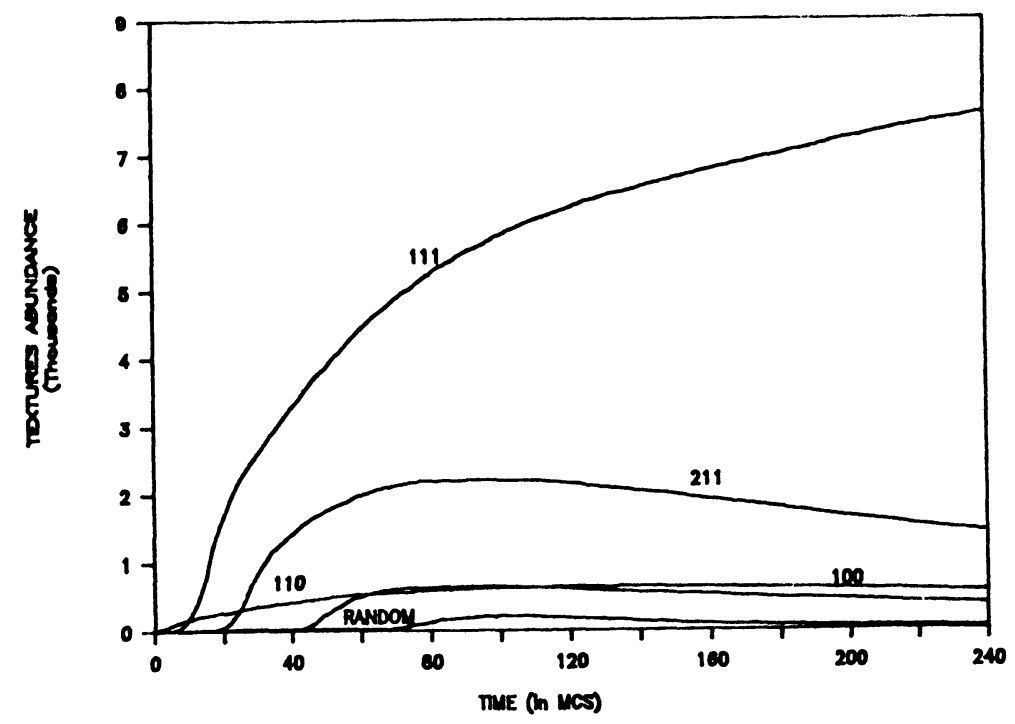

Figure 5 Texture development obtained during simulation of recrystallization in low-carbon steel. 


\section{CONCLUSIONS}

Transformation of texture during annealing is being simulated by modelling the processes of nucleation and grain growth. The framework of simulation described above can be used to model the texture development acconding to the oriented nucleation theory and to the theory of the oriented growth as well. The oriented nucleation theory is modelled by deleting all texture related differences from the grain boundary energy table. This results in continuous nucleation and final saturation of all sites. The oriented growth theory is modelled by assuming a random orientation distribution of nuclei and by assigning the same stored energy to each texture component.

The determination of the grain boundary energy or mobility in polycrystalline materials which have to be used in growth simulation is a rather difficult task since the theoretical predictions for polycrystalline materials are not yet of any practical value, and experimental data are rather uncertain and incomplete.

Similarly the selection of the location of the nucleation sites and their orientations could be made more realistic if the simulation is made for materials having well-known structure.

\section{REFERENCES}

1. D.J. Srolovitz, M.P. Anderson, G.S. Grest and P.S. Sahni, Acta Met. 32, 721 (1984)

2. D.J. Srolovitz, G.S. Grest and M.P. Anderson, Acta Met. 34, 1833 (1986)

3. D.J. Srolovitz, G.S. Grest, M.P. Anderson and A.D. Rollet, Acta Met. 36, 2115 (1988)

4. I. Dillamore and H. Katoh, Metal Science, 8, 21 (1974)

5. W.B. Hutchinson, International Metals Reviews 29, 1 (1984)

6. H. Takechi, Trans. Met. Soc. AME 56, 242 (1968) 